\title{
Solvability of second-order m-point difference equation boundary value problems on infinite intervals
}

\author{
Changlong Yu*, Jufang Wang, Yanping Guo, Surong Miao \\ College of Sciences, Hebei University of Science and Technology, Shijiazhuang, 050018, Hebei, P. R. China. \\ Communicated by A. Atangana
}

\begin{abstract}
In this paper, we study second-order m-point difference boundary value problems on infinite intervals

$$
\left\{\begin{array}{l}
\Delta^{2} x(k-1)+f(k, x(k), \Delta x(k-1))=0, k \in N, \\
x(0)=\sum_{i=1}^{m-2} \alpha_{i} x\left(\eta_{i}\right), \lim _{k \rightarrow \infty} \Delta x(k)=0
\end{array}\right.
$$

where $N=\{1,2, \cdots\}, f: N \times R^{2} \rightarrow R$ is continuous, $\alpha_{i} \in R, \sum_{i=1}^{m-2} \alpha_{i} \neq 1, \eta_{i} \in N, 0<\eta_{1}<\eta_{2}<\cdots<\infty$ and

$$
\Delta x(k)=x(k+1)-x(k)
$$

the nonlinear term is dependent in a difference of lower order on infinite intervals. By using Leray-Schauder continuation theorem, the existence of solutions are investigated. Finally, we give one example to demonstrate the use of the main result. (C)2017 All rights reserved.
\end{abstract}

Keywords: Difference equation, boundary value problem, Leray-Schauder continuation theorem, infinite interval. 2010 MSC: 34B15, 34B16, 34B18, 34G20.

\section{Introduction}

Boundary value problems on infinite intervals originated in the field of the applied mathematics and physics. In recent years, boundary value problems (BVPs) on infinite intervals have received much attention mainly due to their important applications in the study of plasma physics, in analyzing the heat transfer in radial flow between circular disks, in the study of the unsteady flow of a gas through semiinfinite porous medium, and in an analysis of the mass transfer on a rotating disk in non-Newtonian fluid, see $[13,20]$ and references therein. Some works and various techniques dealing with this kind of boundary value problems, such as different kinds of fixed point theorem, upper and lower solution

\footnotetext{
*Corresponding author

Email addresses: changlongyu@126.com (Changlong Yu), wangjufang1981@126.com (Jufang Wang), guoyanping65@126.com (Yanping Guo)

doi:10.22436/jnsa.010.11.11
} 
techniques, topological degree theorem and coincidence degree theorem are used to discuss second-order or $n$-th order boundary value problems on a half-line (Dirichlet problems, periodic, impulsive system, time delay and so on), see $[6,8-11,14,16,19]$ and the references therein.

In fact, differential equations in actual production and scientific research are extremely complex. In many cases, it is difficult to get the analytic solutions and even to get the analytical expressions. To solve the problem, we need to consider its approximate solutions or study on the properties of solutions. It requires the discretization of differential equations, so difference equations is very important and practical. Therefore, many scholars devote to study difference equation boundary value problems. Recently, boundary value problems for difference equations on infinite intervals have been considered widely, such as modern medical biology mathematics, economics, physics, chemistry and so on, and there are some excellent results on the existence of solutions, see $[1,2,4,5,7,12,17,18]$ and the references therein. However, to our knowledge, the theory of difference equation boundary value problems on infinite interval is rather less, there are still lots of work and research that should be done.

In [3], Agarwal and Regan studied the existence of non-negative solutions for second order difference boundary value problems on infinite intervals

$$
\left\{\begin{array}{l}
\Delta^{2} x(i-1)+f(i, x(i))=0, \\
x(0)=0, \quad \lim _{i \rightarrow \infty} x(\mathfrak{i})=0 .
\end{array}\right.
$$

In [15], Lian et al. used the Schauder fixed point theorem and upper and lower solution technique to study unbounded positive solutions for second-order discrete boundary value problems on infinite intervals

$$
\left\{\begin{array}{l}
-\Delta^{2} x_{k-1}=f\left(k, x_{k}, \Delta x_{k-1}\right), \quad k \in N, \\
x_{0}-a \Delta x_{0}=B, \quad \Delta x_{\infty}=C .
\end{array}\right.
$$

Motivated by the work above, in this paper, we consider the existence of solutions for second-order m-point difference boundary value problems on infinite intervals

$$
\left\{\begin{array}{l}
\Delta^{2} x(k-1)+f(k, x(k), \Delta x(k-1))=0, k \in N \\
x(0)=\sum_{i=1}^{m-2} \alpha_{i} x\left(\eta_{i}\right), \lim _{k \rightarrow \infty} \Delta x(k)=0
\end{array}\right.
$$

where $N=\{1,2, \cdots\}, f: N \times R^{2} \rightarrow R$ is continuous, $\alpha_{i} \in R, \sum_{i=1}^{m-2} \alpha_{i} \neq 1, \alpha_{i}$ have the same signal, $\eta_{i} \in N, 0<\eta_{1}<\eta_{2}<\cdots<\infty$ and $\Delta x(k)=x(k+1)-x(k)$.

We set

$$
P=\sum_{j=1}^{\infty} p(j), \quad P_{1}=\sum_{j=1}^{\infty} j p(j), \quad Q=\sum_{j=1}^{\infty} q(j),
$$

and we suppose $\alpha_{i}, i=1,2, \ldots, m-2$ are the same signal in this paper and we always assume $\alpha=\sum_{i=1}^{m-2} \alpha_{i}$.

In this paper, we always assume the following conditions hold:

$\left(C_{1}\right) f: N \times R^{2} \rightarrow R$ is continuous. For each $r>0$, there exists $\varphi_{r}(k) \in l^{1}$ with $k \varphi_{r}(k) \in l^{1}, \varphi_{r}(k)>0$ such that $\max \{|u|,|v|\} \leqslant r$ implies $|f(k, u, v)| \leqslant \varphi_{r}(k)$, for each $k \in N$.

$\left(C_{2}\right) f: N \times R^{2} \rightarrow R$ is continuous, there exist $p(k), q(k), r(k) \in l^{1}$ with $k p(k), k q(k), k r(k) \in l^{1}$, such that for each $k \in N,(u, v) \in R^{2}$ implies $|f(k, u, v)| \leqslant p(k)|u|+q(k)|v|+r(k)$.

We deal with the existence of solutions for BVP (1.1) by using the Lerday-Schauder continuation theorem and obtain the result which extends and improves the known results. 


\section{Preliminary results}

Let $N_{0}$ be the set of all nonnegative integers and $S$ be the space of sequence, that is $x \in S, x=$ $\{x(k)\}_{k \in N_{0}}$. If $x(k) \leqslant y(k)$ for all $k \in N_{0}$, we call $x \leqslant y$. Consider the space

$$
S_{\infty}=\left\{x \in S: \quad \lim _{k \rightarrow \infty} x(k) \text { and } \lim _{k \rightarrow \infty} \Delta x(k) \text { exist }\right\},
$$

with the norm $\|x\|=\max \left\{\|x\|_{\infty},\|\Delta x\|_{\infty}\right\}$, where $\|.\|_{\infty}$ is supremum norm on the infinite intervals. Obviously, $\left(S_{\infty},\|.\|_{\infty}\right)$ is a Banach space. In addition, this paper also involves space $l^{1}$ and for all $x \in l^{1}$, we have $\|x\|_{l^{1}}=\sum_{k=1}^{\infty}|x(k)|$.

Lemma 2.1. Let $v=\{v(k)\}_{k \in N}, \sum_{k=1}^{\infty} v(k)<\infty$ and $\sum_{k=1}^{\infty} k v(k)<\infty$, then the BVP

$$
\left\{\begin{array}{l}
\Delta^{2} x(k-1)+v(k)=0, \quad k \in N \\
x(0)=\sum_{i=1}^{m-2} \alpha_{i} x\left(\eta_{i}\right), \quad \lim _{k \rightarrow \infty} \Delta x(k)=0
\end{array}\right.
$$

has a unique solution. Moreover, this unique solution can be expressed in the form

$$
x(k)=\sum_{j=1}^{\infty} G(k, j) v(j),
$$

where $G(k, j)$ is defined by

$$
G(k, j)=\frac{1}{\Lambda} \begin{cases}j, & j \leqslant \eta_{1}, j \leqslant k, \\ \sum_{i=1}^{m-2} \alpha_{i} j+\Lambda k, & j \leqslant \eta_{1}, j>k, \\ \sum_{a=1}^{i} \alpha_{a} \eta_{a}+\sum_{a=i+1}^{m-2} \alpha_{a} j+\Lambda j, & 0<\eta_{i}<j \leqslant \eta_{i+1}, j \leqslant k, i=1,2, \ldots, m-3, \\ \sum_{a=1}^{i} \alpha_{a} \eta_{a}+\sum_{a=i+1}^{m-2} \alpha_{a} j+\Lambda k, & 0<\eta_{i}<j \leqslant \eta_{i+1}, j>k, i=1,2, \ldots, m-3, \\ \sum_{i=1}^{m-2} \alpha_{i} \eta_{i}+\Lambda j, & \eta_{m-2}<j, j \leqslant k, \\ \sum_{i=1}^{m-2} \alpha_{i} \eta_{i}+\Lambda k, & \eta_{m-2}<j, j>k .\end{cases}
$$

Here $\Lambda=1-\sum_{i=1}^{m-2} \alpha_{i}$

Proof. Since $\sum_{k=1}^{\infty} v(k)<\infty$ and $\sum_{k=1}^{\infty} k v(k)<\infty$, we obtain that

$$
x(k)=x(0)+\sum_{i=1}^{k} \sum_{j=i}^{\infty} v(j)
$$

Since $x(0)=\sum_{i=1}^{m-2} \alpha_{i} x\left(\eta_{i}\right)$, from (2.2), we obtain that

$$
x(0)=\frac{1}{1-\sum_{i=1}^{m-2} \alpha_{i}}\left[\sum_{i=1}^{m-2} \alpha_{i} \sum_{j=1}^{\eta_{1}} j v(j)+\sum_{i=1}^{m-3} \sum_{j=\eta_{i}+1}^{\eta_{i+1}}\left(\sum_{a=i+1}^{m-2} \alpha_{a} j+\sum_{a=1}^{i} \alpha_{a} \eta_{a}\right) v(j)+\sum_{i=1}^{m-2} \alpha_{i} \eta_{i} \sum_{j=\eta_{m-2}+1}^{\infty} v(j)\right] .
$$


The unique solution of (2.1) can be stated by

$$
\begin{aligned}
x(k)= & \frac{1}{1-\sum_{i=1}^{m-2} \alpha_{i}}\left[\sum_{i=1}^{m-2} \alpha_{i} \sum_{j=1}^{\eta_{1}} j v(j)+\sum_{i=1}^{m-3} \sum_{j=\eta_{i}+1}^{\eta_{i+1}}\left(\sum_{a=i+1}^{m-2} \alpha_{a} j+\sum_{a=1}^{i} \alpha_{a} \eta_{a}\right) v(j)+\sum_{i=1}^{m-2} \alpha_{i} \eta_{i} \sum_{j=\eta_{m-2}+1}^{\infty} v(j)\right] \\
& +\sum_{j=1}^{k} j v(j)+\sum_{j=k+1}^{\infty} k v(j) .
\end{aligned}
$$

For $0 \leqslant k \leqslant \eta_{1}$, the unique solution of (2.1) can be stated by

$$
\begin{aligned}
x(k)= & \frac{1}{1-\sum_{i=1}^{m-2} \alpha_{i}} \sum_{j=1}^{k} j v(j)+\sum_{j=k+1}^{\eta_{1}}\left(\frac{\sum_{i=1}^{m-2} \alpha_{i} j}{1-\sum_{i=1}^{m-2} \alpha_{i}}+k\right) v(j) \\
& +\sum_{i=1}^{m-3} \sum_{j=\eta_{i}+1}^{\eta_{i+1}}\left(\frac{\sum_{a=i+1}^{m-2} \alpha_{a} j+\sum_{a=1}^{i} \alpha_{a} \eta_{a}}{1-\sum_{i=1}^{m-2} \alpha_{i}}+k\right) v(j)+\sum_{j=\eta_{m-2}+1}^{\infty}\left(\frac{\sum_{i=1}^{m-2} \alpha_{i} \eta_{i}}{1-\sum_{i=1}^{m-2} \alpha_{i}}+k\right) v(j) .
\end{aligned}
$$

For $\eta_{i}<k \leqslant \eta_{i+1}, 1 \leqslant i \leqslant m-3$, the unique solution of (2.1) can be stated by

$$
\begin{aligned}
& x(k)=\frac{1}{1-\sum_{i=1}^{m-2} \alpha_{i}} \sum_{j=1}^{\eta_{1}} j v(j)+\sum_{b=1}^{i-1} \sum_{j=\eta_{b}+1}^{\eta_{b+1}}\left(\frac{\sum_{a=b+1}^{m-2} \alpha_{a} j+\sum_{a=1}^{b} \alpha_{a} \eta_{a}}{1-\sum_{i=1}^{m-2} \alpha_{i}}+j\right) v(j) \\
& +\sum_{j=\eta_{i}+1}^{k}\left(\frac{\sum_{a=i+1}^{m-2} \alpha_{a} j+\sum_{a=1}^{i} \alpha_{a} \eta_{a}}{1-\sum_{i=1}^{m-2} \alpha_{i}}+j\right) v(j)+\sum_{j=k+1}^{\eta_{i+1}}\left(\frac{\sum_{a=i+1}^{m-2} \alpha_{a} j+\sum_{a=1}^{i} \alpha_{a} \eta_{a}}{1-\sum_{i=1}^{m-2} \alpha_{i}}+k\right) v(j) \\
& +\sum_{b=i+1}^{m-3} \sum_{j=\eta_{b}+1}^{\eta_{b+1}}\left(\frac{\sum_{a=1}^{b} \alpha_{a} \eta_{a}+\sum_{a=b+1}^{m-2} \alpha_{a} j}{1-\sum_{i=1}^{m-2} \alpha_{i}}+k\right) v(j)+\sum_{j=\eta_{m-2}+1}^{\infty}\left(\frac{\sum_{i=1}^{m-2} \alpha_{i} \eta_{i}}{1-\sum_{i=1}^{m-2} \alpha_{i}}+k\right) v(j) .
\end{aligned}
$$

For $\eta_{m-2}<k<\infty$, the unique solution of (2.1) can be stated by

$$
\begin{aligned}
x(k)= & \frac{1}{1-\sum_{i=1}^{m-2} \alpha_{i}} \sum_{j=1}^{\eta_{1}} j v(j)+\sum_{b=1}^{m-3} \sum_{j=\eta_{b}+1}^{\eta_{b+1}}\left(\frac{\sum_{a=b+1}^{m-2} \alpha_{a} j+\sum_{a=1}^{b} \alpha_{a} \eta_{a}}{1-\sum_{i=1}^{m-2} \alpha_{i}}+j\right) v(j) \\
& +\sum_{j=\eta_{m-2}+1}^{k}\left(\frac{\sum_{i=1}^{m-2} \alpha_{i} \eta_{i}}{1-\sum_{i=1}^{m-2} \alpha_{i}}+j\right) v(j)+\sum_{j=k+1}^{\infty}\left(\frac{\sum_{i=1}^{m-2} \alpha_{i} \eta_{i}}{1-\sum_{i=1}^{m-2} \alpha_{i}}+k\right) v(j) .
\end{aligned}
$$

We note $\Lambda=1-\sum_{i=1}^{m-2} \alpha_{i}$, and 


$$
G(k, j)=\frac{1}{\Lambda} \begin{cases}j, & j \leqslant \eta_{1}, j \leqslant k, \\ \sum_{i=1}^{m-2} \alpha_{i} j+\Lambda k, & j \leqslant \eta_{1}, j>k, \\ \sum_{a=1}^{i} \alpha_{a} \eta_{a}+\sum_{a=i+1}^{m-2} \alpha_{a} j+\Lambda j, & 0<\eta_{i}<j \leqslant \eta_{i+1}, j \leqslant k, i=1,2, \ldots, m-3, \\ \sum_{a=1}^{i} \alpha_{a} \eta_{a}+\sum_{a=i+1}^{m-2} \alpha_{a} j+\Lambda k, & 0<\eta_{i}<j \leqslant \eta_{i+1}, j>k, i=1,2, \ldots, m-3, \\ \sum_{i=1}^{m-2} \alpha_{i} \eta_{i}+\Lambda j, & \eta_{m-2}<j, j \leqslant k, \\ \sum_{i=1}^{m-2} \alpha_{i} \eta_{i}+\Lambda k, & \eta_{m-2}<j, j>k .\end{cases}
$$

Therefore, the unique solution of $(2.1)$ is $x(k)=\sum_{j=1}^{\infty} G(k, j) v(j)$, which completes the proof.

Remark 2.2. Obviously $G(k, j)$ satisfies the properties of a Green function, so we call $G(k, j)$ the Green function of the corresponding homogeneous multipoint BVP of (2.1) on infinite intervals.

Lemma 2.3. For all $k, j \in N$, it holds that

$$
|G(k, j)| \leqslant \begin{cases}j, & \sum_{i=1}^{m-2} \alpha_{i}<0, \\ \frac{j}{\Lambda}, & 0 \leqslant \sum_{i=1}^{m-2} \alpha_{i}<1, \\ \max \left\{\frac{\sum_{i=1}^{m-2} \alpha_{i} j}{-\Lambda}, \frac{\sum_{i=1}^{m-2} \alpha_{i} \eta_{m-2}}{-\Lambda}\right\}, & \sum_{i=1}^{m-2} \alpha_{i}>1 .\end{cases}
$$

Proof. For each $j \in N, G(k, j)$ is nondecreasing in $k$, we have

$$
\begin{aligned}
\min & \left\{\frac{\sum_{i=1}^{m-2} \alpha_{i} j}{\Lambda}, \frac{\sum_{a=1}^{i} \alpha_{a} \eta_{a}+\sum_{a=i+1}^{m-2} \alpha_{a} j}{\Lambda}, \frac{\sum_{i=1}^{m-2} \alpha_{i} \eta_{i}}{\Lambda}\right\} \\
& \leqslant G(k, j) \leqslant G(j, j) \\
& =\frac{1}{\Lambda} \begin{cases}j, & j \leqslant \eta_{1}, \\
\sum_{a=1}^{i} \alpha_{a} \eta_{a}+\sum_{a=i+1}^{m-2} \alpha_{a} j+\Lambda j, & 0<\eta_{i}<j \leqslant \eta_{i+1}, i=1,2, \cdots, m-3, \\
\sum_{i=1}^{m-2} \alpha_{i} \eta_{i}+\Lambda j, & \eta_{m-2}<j .\end{cases}
\end{aligned}
$$

Further, we have

$$
\begin{array}{ll}
\frac{\sum_{i=1}^{m-2} \alpha_{i} j}{\Lambda} \leqslant G(k, j) \leqslant j, & \sum_{i=1}^{m-2} \alpha_{i}<0 \\
0<\min \left\{\frac{\sum_{i=1}^{m-2} \alpha_{i} j}{\Lambda}, \frac{\sum_{i=1}^{m-2} \alpha_{i} \eta_{1}}{\Lambda}\right\} \leqslant G(k, j) \leqslant \frac{j}{\Lambda^{\prime}}, & 0 \leqslant \sum_{i=1}^{m-2} \alpha_{i}<1, \\
\min \left\{\frac{\sum_{i=1}^{m-2} \alpha_{i} j}{\Lambda}, \frac{\sum_{i=1}^{m-2} \alpha_{i} \eta_{m-2}}{\Lambda}\right\} \leqslant G(k, j) \leqslant j, & \sum_{i=1}^{m-2} \alpha_{i}>1 .
\end{array}
$$

Therefore, this completes the proof. 
Lemma 2.4. For the Green function $\mathrm{G}(\mathrm{k}, \mathrm{j})$, it holds that

$$
\begin{aligned}
\lim _{k \rightarrow \infty} G(k, j) & =\bar{G}(j) \\
& =\frac{1}{\Lambda} \begin{cases}j, & j \leqslant \eta_{1}, \\
\sum_{a=1}^{i} \alpha_{a} \eta_{a}+\sum_{a=i+1}^{m-2} \alpha_{a} j+\Lambda j, & 0<\eta_{i}<j \leqslant \eta_{i+1}, i=1,2, \ldots, m-3, \\
\sum_{i=1}^{m-2} \alpha_{i} \eta_{i}+\Lambda j, & \eta_{m-2}<j .\end{cases}
\end{aligned}
$$

Theorem 2.5 ([10]). Let

$$
M \subset S_{\infty}=\left\{x \in S: \lim _{k \rightarrow \infty} x(k) \text { and } \lim _{k \rightarrow \infty} \Delta x(k) \text { exist }\right\} .
$$

If $\mathrm{M}$ is uniformly bounded, and uniformly convergent on infinite interval, then $\mathrm{M}$ is relatively compact.

\section{Main result}

Consider the space

$$
X=\left\{x \in S_{\infty}: \quad x(0)=\sum_{i=1}^{m-2} \alpha_{i} x\left(\eta_{i}\right), \lim _{k \rightarrow \infty} \Delta x(k)=0\right\},
$$

and define the operator $\mathrm{T}: \mathrm{X} \times[0,1] \rightarrow \mathrm{X}$ by

$$
T(x, \lambda)(k)=\lambda \sum_{j=1}^{\infty} G(k, j) f(j, x(j), \Delta x(j-1)), \quad k \in N .
$$

The main result of this paper is the following.

Lemma 3.1. Let $\left(\mathrm{C}_{1}\right)$ hold. Then, for each $\lambda \in[0,1], \mathrm{T}(\mathrm{x}, \lambda)$ is completely continuous in $\mathrm{X}$.

Proof. First, we show $T$ is well-defined. For each $x \in X$, then there exists $r>0$ such that $\|x\|<r$. For each $\lambda \in[0,1]$, it holds that

$$
\begin{aligned}
T(x, \lambda)(k) & =\lambda \sum_{j=1}^{\infty} G(k, j) f(j, x(j), \Delta x(j-1)) \\
& \leqslant \sum_{j=1}^{\infty}|G(k, j) f(j, x(j), \Delta x(j-1))| \\
& \leqslant \sum_{j=1}^{\infty}|G(k, j)| \varphi_{r}(j)<\infty, \quad k \in N .
\end{aligned}
$$

By the definition of $\mathrm{T}$, we have

$$
\begin{aligned}
|\Delta T(x, \lambda)(k)| & =|(T(x, \lambda)(k+1)-T(x, \lambda)(k))| \\
& =\left|\lambda \sum_{j=1}^{\infty} G(k+1, j) f(j, x(j), \Delta x(j-1))-\lambda \sum_{j=1}^{\infty} G(k, j) f(j, x(j), \Delta x(j-1))\right| \\
& \leqslant \lambda \sum_{j=1}^{\infty}|G(k+1, j)-G(k, j)| \varphi_{r}(j) \\
& <\infty, \quad k \in N .
\end{aligned}
$$

Therefore, $T x \in S_{\infty}$. 
Obviously, $T(x, \lambda)(0)=\sum_{i=1}^{m-2} \alpha_{i} T(x, \lambda)\left(\eta_{i}\right)$, and notice that

$$
\lim _{k \rightarrow \infty} \Delta T(x, \lambda)(k)=\lim _{k \rightarrow \infty} \lambda \sum_{j=k+1}^{\infty} f(j, x(j), \Delta x(j-1))=0,
$$

so we can get $T x \in X$.

Second, we claim that $T(x, \lambda)$ is completely continuous in $X$, that is, for each $\lambda \in(0,1), T(x, \lambda)$ is continuous in $X$ and maps a bounded subset of $X$ into a relatively compact set.

For each $x_{n} \in X, x_{n} \rightarrow x$ as $n \rightarrow \infty$. Next, we prove that for each $\lambda \in(0,1), T\left(x_{n}, \lambda\right) \rightarrow T(x, \lambda)$ as $\mathrm{n} \rightarrow \infty$ in $\mathrm{X}$. By condition $\left(\mathrm{C}_{1}\right)$, we have

$$
\begin{aligned}
& \left|\sum_{j=1}^{\infty} G(k, j) f\left(j, x_{n}(j), \Delta x_{n}(j-1)\right)-\sum_{j=1}^{\infty} G(k, j) f(j, x(j), \Delta x(j-1))\right| \\
& \leqslant\left|\sum_{j=1}^{\infty} G(k, j)\left(f\left(j, x_{n}(j), \Delta x_{n}(j-1)\right)-f(j, x(j), \Delta x(j-1))\right)\right| \\
& \leqslant 2 \sum_{j=1}^{\infty}|\bar{G}(j)| \varphi_{r_{0}}(j), \quad \text { as } n \rightarrow \infty,
\end{aligned}
$$

where $r_{0}>0$ is a real number, such that $\max _{n \in N}\left\{\left\|x_{n}\right\|,\|x\|\right\}=r_{0}$, we have

$$
\left|T\left(x_{n}, \lambda\right)(k)-T(x, \lambda)(k)\right| \leqslant \sum_{j=1}^{\infty}|G(k, j)| f\left(j, x_{n}(j), \Delta x_{n}(j-1)\right)-f(j, x(j), \Delta x(j-1)) \mid \rightarrow 0, \quad \text { as } n \rightarrow \infty,
$$

and

$$
\left|\Delta T\left(x_{n}, \lambda\right)(k)-\Delta T(x, \lambda)(k)\right| \leqslant \sum_{j=1}^{\infty}\left|f\left(j, x_{n}(j), \Delta x_{n}(j-1)\right)-f(j, x(j), \Delta x(j-1))\right| \rightarrow 0, \quad \text { as } n \rightarrow \infty .
$$

Therefore, $\mathrm{T}$ is continuous.

Finally, we claim that $T$ is compact set, that is, $T$ maps a bounded subset of $X$ into a relatively compact set. Let $B \subset X$ be a bounded subset. For each $x \in B,\|x\|<r$, there exists $r>0$, we have

$$
\begin{aligned}
\|T(x, \lambda)(k)\|_{\infty}=\sup _{k \in N_{0}}|T(x, \lambda)(k)| & =\sup _{k \in N_{0}}\left|\lambda \sum_{j=1}^{\infty} G(k, j) f(j, x(j), \Delta x(j-1))\right| \\
& \leqslant \sum_{j=1}^{\infty}|G(k, j)| \varphi_{r}(k)<\infty, \quad(k \rightarrow \infty) .
\end{aligned}
$$

Similarly, we have

$$
\begin{aligned}
\|\Delta T(x, \lambda)(k)\|_{\infty}=\sup _{k \in N_{0}}|\Delta T(x, \lambda)(k)| & =\sup _{k \in N_{0}}\left|\sum_{j=k+1}^{\infty} f(j, x(j), \Delta x(j-1))\right| \\
& \leqslant \sup _{k \in N_{0}} \sum_{j=k+1}^{\infty} \varphi_{r}(j)<\infty, \quad(k \rightarrow \infty) .
\end{aligned}
$$

Therefore, TB is bounded. At the same time, we obtain that

$$
\begin{aligned}
\left|\left(T(x, \lambda)(k)-\lim _{k \rightarrow \infty} T(x, \lambda)(k)\right)\right| & =\lambda\left|\sum_{j=1}^{\infty}(G(k, j)-\bar{G}(j)) f(j, x(j), \Delta x(j-1))\right| \\
& \leqslant \sum_{j=1}^{\infty}|G(k, j)-\bar{G}(j)| \varphi_{r}(j) \rightarrow 0, \quad(k \rightarrow \infty),
\end{aligned}
$$

and 


$$
\begin{aligned}
\left|\Delta\left(T(x, \lambda)(k)-\lim _{k \rightarrow \infty} \Delta T(x, \lambda)(k)\right)\right| & =|\Delta T(x, \lambda)(k)| \\
& \leqslant \sum_{j=k+1}^{\infty}|f(j, x(j), \Delta x(j-1))| \\
& \leqslant \sum_{j=k+1}^{\infty} \varphi_{r}(j) \rightarrow 0, \quad(k \rightarrow \infty) .
\end{aligned}
$$

So, TB is uniformly convergent on infinity. Thus, by Theorem $2.5, T(\cdot, \lambda): X \times[0,1] \rightarrow X$ is completely continuous on infinite intervals.

Theorem 3.2. Let $\left(\mathrm{C}_{1}\right)$ and $\left(\mathrm{C}_{2}\right)$ hold. Then BVP (1.1) has at least one solution provided:

$$
\begin{aligned}
& \eta_{m-2} P+P_{1}+Q<1, \quad \alpha<0, \\
& \frac{\alpha \eta_{m-2}}{1-\alpha} P+P_{1}+Q<1, \quad 0 \leqslant \alpha<1, \\
& \max \left\{\frac{\alpha \eta_{m-2}}{\alpha-1} P+P_{1}+Q, \frac{\alpha \eta_{m-2}}{\alpha-1} P+\frac{\alpha}{\alpha-1} P_{1}\right\}<1, \quad \alpha>1 .
\end{aligned}
$$

Proof. In view of Lemma 2.1, it is clear that $x \in X$ is a solution of the BVP (1.1) if and only if $x$ is a fixed point of $T(\cdot, 1)$. Clearly, $T(x, 0)=0$ for each $x \in X$. If for each $\lambda \in[0,1]$, the fixed points $T(\cdot, \lambda)$ in $X$ belong to a closed ball of $X$ independent of $\lambda$, then the Leray-Schauder continuation theorem completes the proof. We have known $T(\cdot, \lambda)$ is completely continuous by Lemma 3.1. Next, we show that the fixed point of $T(\cdot, \lambda)$ has a priori bound $M$ independently of $\lambda$. Assume $x=T(x, \lambda)$ and set

$$
Q_{1}=\sum_{j=1}^{\infty} j q(j), \quad R=\sum_{j=1}^{\infty} r(j), \quad R_{1}=\sum_{j=1}^{\infty} j r(j) .
$$

Case 1: $(\alpha<0)$. For any $x \in X, k \in N$, we have

$$
\begin{aligned}
|x(k)|=\left|\sum_{j=1}^{k} \Delta x(j-1)+x(0)\right| & =\left|\sum_{j=1}^{k} \Delta x(j-1)+\frac{\sum_{i=1}^{m-2} \alpha_{i}}{1-\sum_{i=1}^{m-2} \alpha_{i}} \sum_{j=1}^{\eta_{i}} \Delta x(j-1)\right| \\
& \leqslant k\|\Delta x\|_{\infty}+\left|\frac{\alpha}{1-\alpha}\right| \eta_{m-2}\|\Delta x\|_{\infty} \\
& \leqslant\left(k+\eta_{m-2}\right)\|\Delta x\|_{\infty}, \quad k \in N,
\end{aligned}
$$

and so it holds that

$$
\begin{aligned}
\|\Delta x\|_{\infty} \leqslant\|\lambda f(j, x(j), \Delta x(j-1))\|_{l^{1}} & \leqslant\|f(j, x(j), \Delta x(j-1))\|_{l^{1}} \\
& \leqslant\|p(j)|x(j)|+q(j)|\Delta x(j-1)|+r(j)\|_{l^{1}} \\
& \leqslant\left(\eta_{m-2} P+P_{1}+Q\right)\|\Delta x\|_{\infty}+R,
\end{aligned}
$$

therefore,

$$
\|\Delta x\|_{\infty} \leqslant \frac{R}{1-\eta_{m-2} P-P_{1}-Q}:=\Delta M_{1}
$$

At the same time, we have

$$
\begin{aligned}
|x(k)| \leqslant\left|\lambda \sum_{j=1}^{\infty} G(k, j) f(j, x(j), \Delta x(j-1))\right| & \leqslant \sum_{j=1}^{\infty}|j f(j, x(j), \Delta x(j-1))| \\
& \leqslant \sum_{j=1}^{\infty}|j[p(j) x(j)+q(j) \Delta x(j-1)+r(j)]|
\end{aligned}
$$




$$
\begin{aligned}
& \leqslant P_{1}\|x(j)\|_{\infty}+Q_{1}\|\Delta x(j-1)\|_{\infty}+R_{1} \\
& \leqslant P_{1}\|x\|_{\infty}+Q_{1} \Delta M_{1}+R_{1}, \quad k \in N
\end{aligned}
$$

and so

$$
\|x\|_{\infty} \leqslant \frac{Q_{1} \Delta M_{1}+R_{1}}{1-P_{1}}:=M_{1} .
$$

Set $M=\max \left\{M_{1}, \Delta M_{1}\right\}$, which is independent of $\lambda$, so $\|x\| \leqslant M$.

Case 2: $(0 \leqslant \alpha<1)$. For any $x \in X, k \in N$, we have

$$
|x(k)|=\left|\frac{\sum_{i=1}^{m-2} \alpha_{i}}{1-\sum_{i=1}^{m-2} \alpha_{i}} \sum_{j=1}^{\eta_{i}} \Delta x(j-1)+\sum_{j=1}^{k} \Delta x(j-1)\right| \leqslant\left(\frac{\alpha \eta_{m-2}}{1-\alpha}+k\right)\|\Delta x\|_{\infty}, \quad k \in N .
$$

In the same way as for Case 1 , we can get

$$
\begin{aligned}
& \|\Delta x\|_{\infty} \leqslant \frac{(1-\alpha) R}{(1-\alpha)\left(1-P_{1}-Q\right)-\alpha \eta_{m-2} P}:=\Delta M_{2}, \\
& \|x\|_{\infty} \leqslant \frac{Q_{1} \Delta M_{1}+R_{1}}{1-P_{1}}:=M_{2} .
\end{aligned}
$$

Set $M=\max \left\{M_{2}, \Delta M_{2}\right\}$, which is independent of $\lambda$ and is what we need, so $\|x\| \leqslant M$.

Case 3: $(\alpha>1)$. For any $x \in X, k \in N$, we have

$$
|x(k)|=\left|x(0)+\sum_{j=1}^{k} \Delta x(j-1)\right| \leqslant\left(\frac{\alpha \eta_{m-2}}{\alpha-1}+k\right)\|\Delta x\|_{\infty}, \quad k \in N .
$$

Similarly, we obtain

$$
\|\Delta x\|_{\infty} \leqslant \frac{(\alpha-1) R}{(\alpha-1)\left(1-P_{1}-Q\right)-\alpha \eta_{m-2} P}:=\Delta M_{3}
$$

and

$$
\begin{aligned}
|x(k)| & \leqslant \sum_{j=1}^{\infty}|G(k, j) f(j, x(j), \Delta x(j-1))| \\
& \leqslant \sum_{j=1}^{\infty}\left|\frac{\alpha j}{\alpha-1} f(j, x(j), \Delta x(j-1))\right|+\sum_{j=\eta}^{\infty}\left|\frac{\alpha \eta_{m-2}}{\alpha-1} f(j, x(j), \Delta x(j-1))\right| \\
& \leqslant \frac{\alpha}{\alpha-1}\left(P_{1}\|x\|_{\infty}+Q_{1} \Delta M_{3}+R_{1}\right)+\frac{\alpha \eta_{m-2}}{\alpha-1}\left(P\|x\|_{\infty}+Q \Delta M_{3}+R\right),
\end{aligned}
$$

that is,

$$
\|x\|_{\infty} \leqslant \frac{\alpha\left(Q_{1} \Delta M_{3}+R_{1}\right)+\alpha \eta_{m-2}\left(Q_{1} \Delta M_{3}+R\right)}{\alpha-1-\alpha\left(P_{1}+\eta_{m-2} P\right)}:=M_{3}
$$

Set $M=\max \left\{M_{3}, \Delta M_{3}\right\}$ and this is what we need. Hence, BVP (1.1) has at least one solution.

\section{Example}

Example 4.1. Consider the following second-order four-point difference equation BVP on infinite intervals

$$
\left\{\begin{array}{l}
\Delta^{2} x(k-1)+\frac{\sin (x(k))}{10^{2} k !}+\frac{\arctan (\Delta x(k-1))+1}{3^{k}}=0, \quad k \in N, \\
x(0)=\frac{1}{10} x(10)+\frac{1}{10} x(100), \quad \lim _{k \rightarrow \infty} \Delta x(k)=0,
\end{array}\right.
$$

where $m=4, \alpha_{1}=\alpha_{2}=\frac{1}{10}, \eta_{1}=10, \eta_{2}=100, f(t, u, v)=\frac{\sin (x(k))}{10^{2} k !}+\frac{\arctan (\Delta x(k-1))+1}{3^{k}}$, and $p(k)=$ 
$\frac{1}{10^{2} \mathrm{k} !}, \mathrm{q}(\mathrm{k})=\frac{1}{3^{\mathrm{k}}}, \mathrm{r}(\mathrm{k})=\frac{1}{3^{\mathrm{k}}}$.

Obviously, $f(t, u, v) \leqslant p(k)|u|+q(k)|v|+r(k)$. By a simple calculation, we find that $\left(C_{1}\right)$ and $\left(C_{2}\right)$ hold. Since $\alpha=\alpha_{1}+\alpha_{2}=\frac{1}{5}<1, \frac{\alpha \eta_{2}}{1-\alpha} \mathrm{P}+\mathrm{P}_{1}+\mathrm{Q} \approx 0.96<1$. Hence, by Theorem 3.2, we obtain the BVP (4.1) has at least one solution.

\section{Acknowledgment}

This paper is supported by the Natural Science Foundation of China (11371120), the Natural Science Foundation of Hebei Province (A2014208152) and (A2015208114), the Foundation of Hebei Education Department (QN2016165) and (QN2017063), and the Foundation of Hebei University of Science and Technology (2016ZDYY24).

\section{References}

[1] R. P. Agarwal, M. Bohner, D. O'Regan, Time scale boundary value problems on infinite intervals, Dynamic equations on time scales, J. Comput. Appl. Math., 141 (2002), 27-34. 1

[2] R. P. Agarwal, D. O'Regan, Boundary value problems for general discrete systems on infinite intervals, Comput. Math. Appl., 33 (1997), 85-99. 1

[3] R. P. Agarwal, D. O'Regan, Discrete systems on infinite intervals, Comput. Math. Appl., 35 (1998), 97-105. 1

[4] R. P. Agarwal, D. O'Regan, Existence and approximation of solutions of non-linear discrete systems on infinite intervals, Math. Methods Appl. Sci., 22 (1999), 91-99. 1

[5] R. P. Agarwal, D. O'Regan, Continuous and discrete boundary value problems on the infinite interval: existence theory, Mathematika, 48 (2001), 273-292. 1

[6] R. P. Agarwal, D. O'Regan, Infinite interval problems for differential, difference and integral equations, Kluwer Academic Publishers, Dordrecht, (2001). 1

[7] R. P. Agarwal, D. O'Regan, Nonlinear Urysohn discrete equations on the infinite interval: a fixed-point approach, Advances in difference equations, III, Comput. Math. Appl., 42 (2001), 273-281. 1

[8] R. P. Agarwal, D. O'Regan, Non-linear boundary value problems on the semi-infinite interval: an upper and lower solution approach, Mathematika, 49 (2002), 129-140. 1

[9] B. Ahmad, A. Alsaedi, B. S. Alghamdi, Analytic approximation of solutions of the forced Duffing equation with integral boundary conditions, Nonlinear Anal. Real World Appl., 9 (2008), 1727-1740.

[10] J. V. Baxley, Existence and uniqueness for nonlinear boundary value problems on infinite intervals, J. Math. Anal. Appl., 147 (1990), 122-133. 2.5

[11] Y.-P. Guo, C.-L. Yu, J.-F. Wang, Existence of three positive solutions for m-point boundary value problems on infinite intervals, Nonlinear Anal., 71 (2009), 717-722. 1

[12] G. S. Guseinov, A boundary value problem for second order nonlinear difference equations on the semi-infinite interval, Special issue in honour of Professor Allan Peterson on the occasion of his 60th birthday, Part I, J. Difference Equ. Appl., 8 (2002), 1019-1032. 1

[13] R. E. Kidder, Unsteady flow of gas through a semi-infinite porous medium, J. Appl. Mech., 27 (1957), 329-332. 1

[14] H.-R. Lian, W.-G. Ge, Solvability for second-order three-point boundary value problems on a half-line, Appl. Math. Lett., 19 (2006), 1000-1006. 1

[15] H.-R. Lian, J.-W. Li, R. P. Agarwal, Unbounded solutions of second order discrete BVPs on infinite intervals, J. Nonlinear Sci. Appl., 9 (2016), 357-369. 1

[16] D. O'Regan, Theory of singular boundary value problems, World Scientific Publishing Co., Inc., River Edge, NJ, (1994). 1

[17] Y. Tian, W.-G. Ge, Multiple positive solutions of boundary value problems for second-order discrete equations on the halfline, J. Difference Equ. Appl., 12 (2006), 191-208. 1

[18] Y. Tian, C. C. Tisdell, W.-G. Ge, The method of upper and lower solutions for discrete BVP on infinite intervals, J. Difference Equ. Appl., 17 (2011), 267-278. 1

[19] C.-L. Yu, J.-F. Wang, G.-G. Li, Existence of positive solutions for nth-order integral boundary value problems with $p$ Laplacian operator on infinite interval, J. Hebei Univ. Sci. Technol., 36 (2015), 382-389. 1

[20] L. Zheng, X. Zhang, J. He, Singular nonlinear boundary value problem for transmission process, Science Press, Beijing, (2003). 1 